\title{
Memory Effects Due to Fractional Time Derivative and Integral Space in Diffusion Like Equation Via Haar Wavelets
}

\author{
I. K. Youssef ${ }^{1}$, A. R. A. Ali ${ }^{2}$ \\ ${ }^{1}$ Department of Mathematics, Ain Shams University, Cairo, Egypt \\ ${ }^{2}$ Department of Mathematics, Baghdad University, Baghdad, Iraq \\ Email address: \\ Kaoud22@hotmail.com (I. K. Youssef), adil.r.a@ihcoedu.uobaghdad.edu.iq (A. R. A. Ali)
}

\section{To cite this article:}

I. K. Youssef, A. R. A. Ali. Memory Effects Due to Fractional Time Derivative and Integral Space in Diffusion Like Equation Via Haar Wavelets. Applied and Computational Mathematics. Vol. 5, No. 4, 2016, pp. 177-185. doi: 10.11648/j.acm.20160504.12

Received: August 6, 2016; Accepted: August 15, 2016; Published: September 2, 2016

\begin{abstract}
Memory and hereditary effects due to fractional time derivative are combined with the global behaviours due to space integral term. Haar wavelet operational matrix is adjusted to solve diffusion like equations with time fractional derivative, space derivatives and integral terms. The fractional derivative is understood in the Caputo sense. The memory behaviours is included in all the points of the domain due to the existence of space integral term and the inverse fractional operator treatment and this is ilustrated in error graphs introduced. A general example with four subproblems ranging from the simple classical heat equation to the fractional time diffusion equation with global integral term is proposed and the calculated results are displayed graphically.
\end{abstract}

Keywords: Haar Wavelet, Operational Matrix, Fractional Derivative, Diffusion Like Equation

\section{Introduction}

Due to the developments in the environmental technology many mathematical models had been reformulated to recover the real situations. Integral equations models consider the global behaviors of the physical processes while differential equations models consider the local behaviors. Integrodifferential equations models is a vital step in the developments of mathematical models. Delay differential equations considers the back history of the phenomena under consideration it is a step towards realistic models. Many authors have considered integral or differential or integrodifferential equations with delay parameters. Recently, fractional order differential equations is used in modeling many physical and engineering processes such as anomalous diffusion, complex viscoelasticity and behaviors in mechatronic and biological fields. Fractional order differential equations consider the memory and hereditary effects in addition to the local behaviors. In previous works the authors have studided the dificulties of solving some problems related to the diffusion equation with different methods. In [1] the solution of the simple diffusion equation is considered by the use of with restrictive Chebyshev rational approximation. In
[2] Rektorys considered the diffusion equations with integral terms appears in the non- homogeneous term or in the boundary conditions with the method of discretization in time. In [3] El-Sayed considered the fractional order diffusion wave equation. In [4] Youssef and Shukur considered use the method of lines to construct an approximate solution to a fractional time and space diffusion equation. In [5] Youssef and Shukur use the modified variational iteration method to construct an approximate solution to a fractional time and space diffusion equation. In [6] the authors considered the memory effects due to the existence of fractional time derivatives and a time dependent integral term via Haar wavelets treatment in the form

$$
\frac{\partial^{\alpha} u(x, t)}{\partial t^{\alpha}}=\frac{\partial^{2} u(x, t)}{\partial x^{2}}+\sigma \int_{0}^{t} u(x, s) d s+f(x, t)
$$

Where $0<\alpha \leq 1, t$ and $x$ are arguments; usually $0 \leq t \leq T$ denotes time, $0 \leq x \leq$, and $\sigma=0$ or 1 , with initial condition $u(x, 0)=f(x)$ and boundary conditions $u(0, t)=g_{0}(t), u(1, t)=g_{1}(t)$. The motivation for such equations lies in different branches of physics, in rheology, and especially in the theory of heat conduction when inner heat sources are of special types [2].

In this work the memory and hereditary behaviors are 
considered through fractional derivatives and the global behaviors through considering space integral terms.

$$
\frac{\partial^{\alpha} u(x, t)}{\partial t^{\alpha}}=\frac{\partial^{2} u(x, t)}{\partial x^{2}}+\sigma \int_{0}^{x} u(s, t) d s+f(x, t)
$$

Where the fractional order derivatives is understood in the Caputo sense.

Definition (1): The Caputo time fractional derivative of order $\alpha>0$ of the function $u(x, t)$ is defined by [7,8]:

$$
\frac{\partial^{\alpha} u(x, t)}{\partial t^{\alpha}}=\frac{1}{\Gamma(n-\alpha)} \int_{0}^{t}(t-s)^{n-\alpha-1} \frac{d^{n}}{d s^{n}} u(x, s) d s
$$

where $\alpha \in(n-1, n), n \in N$. If $\alpha \in N$, then this will coincide with the classical partial derivative.

Equation (2) considers the memory effectis in time through the fractional time derivatives and the memory effects in space through the integral term.

The number of publications about the fractional calculus has rapidly increased because of some physical processes as anomalous diffusion, complex viscoelasticity, behavior of mechatronic and biological systems, rheology etc. cannot be described adequately by the classical models [9]. The fractional derivatives is understood in the Caputo sense.

As our treatment in [6] the fractional derivatives appears in equation (2) can be translated to the right hand side as

$$
\frac{\partial u(x, t)}{\partial t}=\frac{\partial^{1-\alpha}}{\partial t^{1-\alpha}}\left(\frac{\partial^{2} u(x, t)}{\partial x^{2}}\right)+\sigma \frac{\partial^{1-\alpha}}{\partial t^{1-\alpha}} \int_{0}^{x} u(s, t) d s+\frac{\partial^{1-\alpha}}{\partial t^{1-\alpha}} f(x, t)
$$

Thus, the memory and hereditary effects due to the fractional time derivatives have enforced to the terms on the right hand side and this well be clear during the numerical calculations.

Due to the developments in computational systems (techniques and devices) numerical methods are considered as the master methods for such problems. Among numerical methods the finite differences $[4,5,7,10,11]$, the weighted residual methods specially the finite element method also spectral methods or combinations of them are heavily used in solving such problems. Recently, the wavelet methods are rapidly used and the Haar wavelet is the simplest known wavelet method.

Haar wavelets are made up of pairs of piecewise constant functions and are mathematically the simplest among all the wavelet families. The Haar wavelet is the only real valued wavelet function which is symmetrical, orthogonal and has a compact support [12]. A good feature of the Haar wavelets is the possibility to integration analytically arbitrary times. The Haar wavelets are very effective for treating singularities, since they can be interpreted as intermediate boundary conditions [13], but the disadvantage of the Haar wavelets is their discontinuity since the derivatives do not exist in the breaking end points and it is not possible to apply the Haar wavelets for solving partial differential equations directly.

Chen and Hsiao [14, 15], who first proposed a Haar product matrix and a coefficient matrix, they derived a Haar operational matrix for the integrals of the Haar function vector and put the application for the Haar analysis into the dynamic systems. The method technique is approximate the highest derivative of the differential equation with finite Haar wavelet series. Then integrate this approximation to get the lower order derivatives in the equation. Many authors use this technique to solve the differential and integral equations [16, $17,18,19,20]$.

\section{Haar Wavelets}

The use of Haar wavelet in solving problems of calculus appears only from 1997, [16]. The technique is described in many publications $[6,14,15]$ and the references cited there. The Haar wavelet family, $h_{n}(x) ; 0 \leq x \leq 1$ is used as bases and are defined as:

$$
h_{0}(x)= \begin{cases}1, & 0<x \leq 1, \\ 0, & \text { otherwise }\end{cases}
$$

the mother wavelets function $h_{1}(t)$ is

$$
h_{1}(x)= \begin{cases}1, & 0 \leq x<\frac{1}{2} \\ -1, & \frac{1}{2} \leq x<1 \\ 0, & \text { otherwise }\end{cases}
$$

and for $n \geq 2$ the Haar wavelet is defined from $h_{1}(x)$ by translation and dilation operations. That is

$$
h_{n}(x)=h_{1}\left(2^{j} x-k\right) ; n \geq 1
$$

Where $n=2^{j}+k, 0 \leq j, 0 \leq k<2^{j}$. The Haar wavelet functions are orthogonal in the sense

$$
\int_{0}^{1} h_{m}(x) h_{n}(x) d x=2^{-j} \delta_{m n}= \begin{cases}2^{-j}, & m=n=2^{-j}+k, \\ 0, & m \neq n\end{cases}
$$

Accordingly, Haar wavelets are independent in the interval $(0,1)$. Wavelet analysis allows representing a function or signal in terms of a set of orthonormal basis functions called wavelets. Haar wavelets are a basis for $L_{2}[0,1]$, for more details you can see [6] and the references there in.

\section{Function Approximation}

It is well known that any function $y(x) \in L_{2}[0,1]$ can be written as

$$
y(x)=\sum_{n=0}^{\infty} c_{n} h_{n}(x)
$$

where the coefficients $c_{n}$ are determined by

$$
c_{n}=2^{j} \int_{0}^{1} y(s) h_{n}(s) d s, n \geq 0
$$

with $n=2^{j}+k, 0 \leq j$, and $0 \leq k<2^{j}$, [6]. Generally, the series in (8) can be truncated to a finite number of terms.

$$
y(x)=\sum_{n=0}^{m-1} c_{n} h_{n}(x)=\boldsymbol{C}_{(m)}^{T} \boldsymbol{h}_{(m)}(x)
$$

Where as usual the coefficient vector $\boldsymbol{C}_{(m)}^{T}$ and the Haar function vector $\boldsymbol{h}_{(m)}(x)$ are define as

$$
\boldsymbol{C}_{(m)}^{T}=\left[c_{0}, c_{1}, \cdots, c_{m-1}\right]
$$




$$
\boldsymbol{h}_{(m)}(x)=\left[h_{0}(x), h_{1}(x), h_{m-1}(x)\right]^{T}
$$

Where $T$ means transpose and $m=2^{j}$.

The first four Haar function vectors which $x=\left[\frac{1}{8}, \frac{3}{8}, \frac{5}{8}, \frac{7}{8}\right]$ can be expressed the following [14]

$$
\begin{gathered}
\boldsymbol{h}_{(4)}(1 / 8)=[1,1,1,0]^{T}, \boldsymbol{h}_{(4)}(3 / 8)=[1,1,-1,0]^{T}, \\
\boldsymbol{h}_{(4)}(5 / 8)=[1,-1,0,1]^{T}, \boldsymbol{h}_{(4)}(7 / 8)=[1,-1,0,1]^{T}
\end{gathered}
$$

this can be written in matrix form as

$$
\begin{aligned}
\boldsymbol{H}_{(4)}=\left[\boldsymbol{h}_{(4)}(1 / 8),\right. & \left.\boldsymbol{h}_{(4)}(3 / 8), \boldsymbol{h}_{(4)}(5 / 8), \boldsymbol{h}_{(4)}(7 / 8)\right] \\
= & {\left[\begin{array}{cccc}
1 & 1 & 1 & 1 \\
1 & 1 & -1 & -1 \\
1 & -1 & 0 & 0 \\
0 & 0 & 1 & -1
\end{array}\right] }
\end{aligned}
$$

Here $\boldsymbol{H}_{(m)}$ denotes the Haar matrix with the components $\boldsymbol{H}(i, l)=\boldsymbol{h}_{\mathrm{i}}\left(x_{l}\right) \quad$ where $x_{l}=\left[\frac{1}{2 \mathrm{~m}}, \frac{3}{2 \mathrm{~m}}, \cdots, \frac{2 \mathrm{~m}-1}{2 \mathrm{~m}}\right]$ are the collecation points. In general, we have

$$
\boldsymbol{H}_{(m)}=\left[\boldsymbol{h}_{(m)}\left(\frac{1}{2 m}\right), \boldsymbol{h}_{(m)}\left(\frac{3}{2 m}\right), \cdots, \boldsymbol{h}_{(m)}\left(\frac{2 m-1}{2 m}\right)\right]
$$

where $\boldsymbol{H}_{(1)}=[1], \boldsymbol{H}_{(2)}=\left[\begin{array}{ll}1 & 1 \\ 1 & -1\end{array}\right]$.

The equation (6) can be rewritten as

$$
h_{n}(x)=\left\{\begin{array}{cc}
1 & \eta_{1} \leq x<\eta_{2} \\
-1 & \eta_{2} \leq x<\eta_{3} \\
0 & \text { otherwise }
\end{array}\right.
$$

where $\eta_{1}=\frac{k}{2^{j}}, \eta_{2}=\frac{k+0.5}{2^{j}}$ and $\eta_{3}=\frac{k+1}{2^{j}}$. Introduce the following notations

$$
\begin{gathered}
P_{n, 1}(x)=\int_{0}^{x} h_{n}(s) d s \\
P_{n, v}(x)=\int_{0}^{x} P_{n, v-1}(s) d s
\end{gathered}
$$

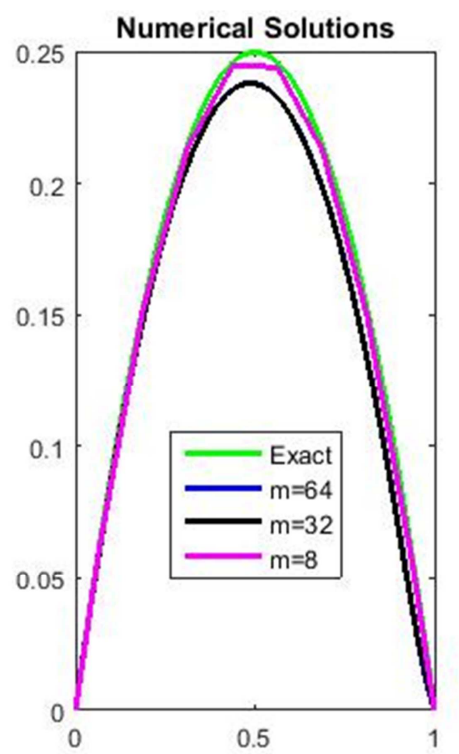

Then The integration of $h_{n}(x)$ can be evaluated analytically using equation (15) and given by

$$
\begin{gathered}
P_{n, 1}(x)=\left\{\begin{array}{cc}
x-\eta_{1} & \eta_{1} \leq x<\eta_{2} \\
\eta_{3}-x & \eta_{2} \leq x<\eta_{3} \\
0 & \text { otherwise }
\end{array}\right. \\
P_{n, 2}(x)=\left\{\begin{array}{cc}
\frac{1}{2}\left(x-\eta_{1}\right)^{2} & \eta_{1} \leq x<\eta_{2} \\
2^{-2 j-2}-\frac{1}{2}\left(\eta_{3}-x\right)^{2} & \eta_{2} \leq x<\eta_{3} \\
2^{-2 j-2} & \eta_{3} \leq x<1 \\
0 & \text { otherwise }
\end{array}\right.
\end{gathered}
$$

and for arbitrary $v$

$$
P_{n, v}(x)=\left\{\begin{array}{cc}
0 & x<\eta_{1} \\
\frac{1}{v !}\left(x-\eta_{1}\right)^{v} & \eta_{1} \leq x<\eta_{2} \\
\left.\frac{1}{v !}\left(x-\eta_{1}\right)^{v}-2\left(x-\eta_{2}\right)^{v}\right\} & \eta_{2} \leq x<\eta_{3} \\
\frac{1}{v !}\left\{\left(x-\eta_{1}\right)^{v}-2\left(x-\eta_{2}\right)^{v}+\left(x-\eta_{3}\right)^{v}\right\} & \eta_{3} \leq x
\end{array}\right.
$$

The integration of the vector $\boldsymbol{h}_{(m)}(x)$ is given by Chen and Hsiao method [14], who first proposed a Haar product matrix and a coefficient matrix

$$
\int_{0}^{\mathrm{x}} \boldsymbol{h}_{(m)}(s) d s=\boldsymbol{P}_{(m)} \boldsymbol{h}_{(m)}(x)
$$

Where $0 \leq x<1$ and $\boldsymbol{P}_{(m)}$ is the $m \times m$ operational matrix. proved that

$$
\boldsymbol{P}_{(m)}=\frac{1}{2 m}\left[\begin{array}{ll}
2 m \boldsymbol{P}_{(m / 2)} & -\boldsymbol{H}_{(m / 2)} \\
\boldsymbol{H}_{(m / 2)}^{-1} & 0
\end{array}\right]
$$

Where $\quad \boldsymbol{P}_{(1)}=\left[\frac{1}{2}\right], \quad \boldsymbol{P}_{(2)}=\frac{1}{4}\left[\begin{array}{ll}2 & -1 \\ 1 & 0\end{array}\right], \quad \boldsymbol{P}_{(4)}=$ $\frac{1}{16}\left[\begin{array}{cccc}8 & -4 & -2 & -2 \\ 4 & 0 & -2 & 2 \\ 1 & 1 & 0 & 0 \\ 1 & -1 & 0 & 0\end{array}\right]$ and so on.

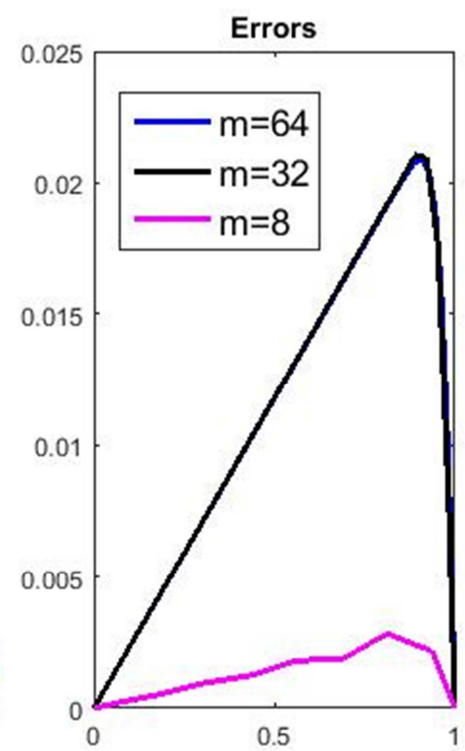

Figure 1. Comparison between the exact solution and the approximate solutions of the partial differential equation for Case 1 equation (41) for $m=8,32,64$ ( $m=16$ is unstable). 


\section{Method of Solution}

Generally, the dominant derivative term appear in the problem can be written as a finite series in the form, [6]

$$
\dot{u}^{\prime \prime}(x, t)=\sum_{n=0}^{m-1} c_{n}\left(t_{s}\right) h_{n}(x)=\boldsymbol{C}_{(m)}^{T} \boldsymbol{h}_{(m)}(x)
$$

The dominant derivative term means the term which contains the highest derivatives in only one term, the dot is used to denote derivatives with respect time and the primes means differentiation with respect to the space $x$.
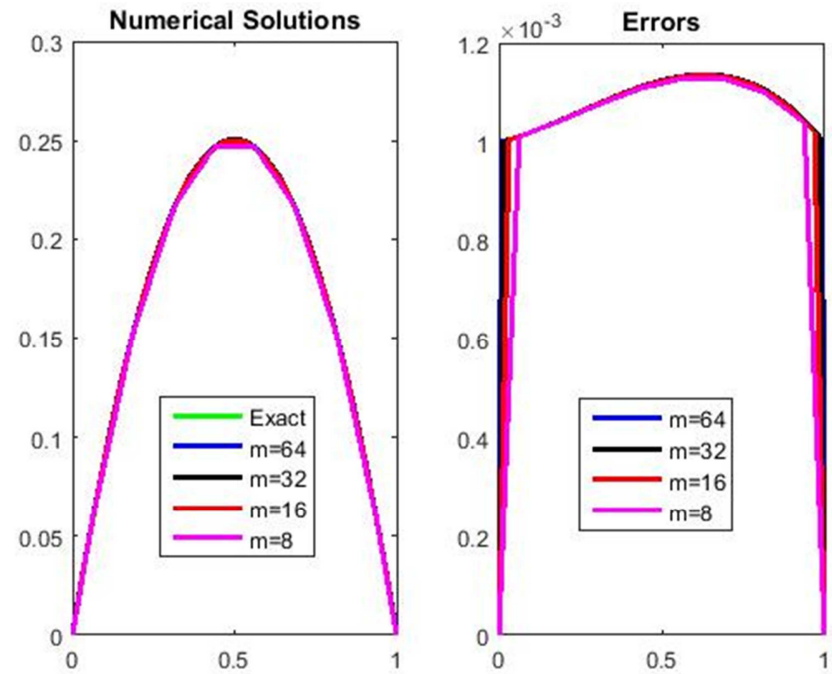

Figure 2. Comparison between the exact solution and the approximate solutions of the partial differential equation for Case 2 equation (44) for $m=8,16,32,64$

divide the time interval $\left[0, T_{\text {fin }}\right]$ into $N$ parts of length $d t$ and expand the highest derivative $\dot{u}^{\prime \prime}(x, t)$ terms of the Haar wavelet as equation (23). Where $t_{s}=s d t, s=1,2, \cdots, N$. with assuming the row vector $\boldsymbol{C}_{(m)}^{T}$ is constant in the subinterval $\left[t_{s}, t_{s+1}\right)$. Integrating formula (23) with respect to $t$ from $t_{s}$ to $t$ and twice with respect to $x$ from 0 to $x$, and using formula (21), the quantities $u^{\prime \prime}(x, t), u^{\prime}(x, t), u(x, t)$ and $\dot{u}(x, t)$ can be expressed as:

$$
\begin{gathered}
u^{\prime \prime}(x, t)=u^{\prime \prime}\left(x, t_{s}\right)+\left(t-t_{s}\right) \boldsymbol{C}_{(m)}^{T} \boldsymbol{h}_{(m)}(x) \\
u^{\prime}(x, t)=u^{\prime}\left(x, t_{s}\right)+u^{\prime}(0, t)-u^{\prime}\left(0, t_{s}\right) \\
+\left(t-t_{s}\right) \boldsymbol{C}_{(m)}^{T} \boldsymbol{P}_{(m)} \boldsymbol{h}_{(m)}(x) \\
u(x, t)=\left(t-t_{s}\right) \boldsymbol{C}_{(m)}^{T} \boldsymbol{P}_{(m)}^{2} \boldsymbol{h}_{(m)}(x)+u\left(x, t_{s}\right) \\
-u\left(0, t_{s}\right)+u(0, t)+x\left[u^{\prime}(0, t)-u^{\prime}\left(0, t_{s}\right)\right] \\
\dot{u}(x, t)=\boldsymbol{C}_{(m)}^{T} \boldsymbol{P}_{(m)}^{2} \boldsymbol{h}_{(m)}(x)+\dot{u}(0, t)+x \dot{u}^{\prime}(0, t)
\end{gathered}
$$

From the boundary conditions, we can get $u\left(0, t_{s}\right)=$ $g_{0}\left(t_{s}\right), u\left(1, t_{s}\right)=g_{1}\left(t_{s}\right), \dot{u}(0, t)=\dot{\mathrm{g}}_{0}(t), \dot{u}(1, t)=\dot{\mathrm{g}}_{1}(t)$, Putting $x=1$ in formula (26) and (27) to get

$$
\begin{gathered}
u^{\prime}(0, t)=u^{\prime}\left(0, t_{s}\right)-\left(t-t_{s}\right) \boldsymbol{C}_{(m)}^{T} \boldsymbol{P}_{(m)}^{2} \boldsymbol{h}_{(m)}(x) \\
+g_{1}(t)-g_{1}\left(t_{s}\right)+g_{0}\left(t_{s}\right)-g_{0}(t)
\end{gathered}
$$

$$
\dot{u}^{\prime}(0, t)=-\boldsymbol{C}_{(m)}^{T} \boldsymbol{P}_{(m)}^{2} \boldsymbol{f}+\dot{\mathrm{g}}_{1}(t)-\dot{\mathrm{g}}_{0}(t)
$$

Where the vector $f$ is defined as $f=[1,0, \cdots, 0]^{T}$. Substituting formula (28) and (29) into equations (24) to (27) and rewrite the results by assuming $x=x_{l}, t=t_{s+1}$ and $d t=\left(t-t_{s}\right)$ to obtain

$$
\begin{gathered}
u^{\prime \prime}\left(x_{l}, t_{s+1}\right)=u^{\prime \prime}\left(x_{l}, t_{s}\right)+d t \boldsymbol{C}_{(m)}^{T} \boldsymbol{h}_{(m)}(x) \\
u^{\prime}\left(x_{l}, t_{s+1}\right)=u^{\prime}\left(x_{l}, t_{s}\right)+d t \boldsymbol{C}_{(m)}^{T} \boldsymbol{P}_{(m)} \boldsymbol{h}_{(m)}(x) \\
-d t \boldsymbol{C}_{(m)}^{T} \boldsymbol{P}_{(m)} \boldsymbol{f} \\
+g_{1}\left(t_{s+1}\right)-g_{1}\left(t_{s}\right)+g_{0}\left(t_{s}\right)-g_{0}\left(t_{s+1}\right) \\
u\left(x_{l}, t_{s+1}\right)=d t \boldsymbol{C}_{(m)}^{T} \boldsymbol{P}_{(m)}^{2} \boldsymbol{h}_{(m)}\left(x_{l}\right)+u\left(x_{l}, t_{s}\right)-g_{0}\left(t_{s}\right) \\
+g_{0}\left(t_{s+1}\right)+x_{l}\left[-d t \boldsymbol{C}_{(m)}^{T} \boldsymbol{P}_{(m)} \boldsymbol{f}+g_{1}\left(t_{s+1}\right)\right. \\
\left.-g_{1}\left(t_{s}\right)+g_{0}\left(t_{s}\right)-g_{0}\left(t_{s+1}\right)\right] \\
\dot{u}\left(x_{l}, t_{s+1}\right)=\boldsymbol{C}_{(m)}^{T} \boldsymbol{P}_{(m)}^{2} \boldsymbol{h}_{(m)}\left(x_{l}\right)+\dot{u}\left(0, t_{s+1}\right) \\
+x_{l}\left[-\boldsymbol{C}_{(m)}^{T} \boldsymbol{P}_{(m)}^{2} \boldsymbol{f}+\dot{\mathrm{g}}_{1}\left(t_{s+1}\right)-\dot{\mathrm{g}}_{0}\left(t_{s+1}\right)\right]
\end{gathered}
$$

and for the space integral term

$$
\begin{array}{r}
\int_{0}^{x} u(s, t) d s=\operatorname{dt} \boldsymbol{C}_{(m)}^{T} \boldsymbol{P}_{(m)}^{3} \boldsymbol{h}_{(m)}\left(x_{l}\right)+\mathrm{x}_{l}\left[u\left(\mathrm{x}_{l}, \mathrm{t}_{s}\right)-\right. \\
\left.\mathrm{g}_{0}\left(\mathrm{t}_{s}\right)+\mathrm{g}_{0}\left(\mathrm{t}_{s+1}\right)\right]+\mathrm{x}_{l}^{2}\left[-\mathrm{dt} \boldsymbol{C}_{(m)}^{T} \boldsymbol{P}_{(m)} \boldsymbol{f}+-\mathrm{g}_{1}\left(\mathrm{t}_{s}\right)+\right. \\
\left.\mathrm{g}_{1}\left(\mathrm{t}_{s+1}\right)+\mathrm{g}_{0}\left(\mathrm{t}_{s}\right)-\mathrm{g}_{0}\left(\mathrm{t}_{s+1}\right)\right]
\end{array}
$$

The fractional derivative $\frac{\partial^{\alpha} u(x, t)}{\partial t^{\alpha}}$ is understood in the Caputo sense defined above. Accordinglly, the fractional derivative $\frac{\partial^{\alpha} u(x, t)}{\partial t^{\alpha}}$ can be rewritten as:

$$
\frac{\partial^{\alpha} u(x, t)}{\partial t^{\alpha}}=\frac{1}{\Gamma(1-\alpha)} \int_{0}^{t}(t-s)^{-\alpha}\left[\boldsymbol{C}_{(m)}^{T} \boldsymbol{P}_{(m)}^{2} \boldsymbol{h}_{(m)}(x)+\right.
$$

$\left.\dot{\mathrm{g}}_{0}\left(t_{s+1}\right)+x_{l}\left[-\boldsymbol{C}_{(m)}^{T} \boldsymbol{P}_{(m)}^{2} \boldsymbol{f}+\dot{\mathrm{g}}_{0}\left(t_{s+1}\right)-\dot{\mathrm{g}}_{0}\left(t_{s+1}\right)\right]\right] d s$

which can be rearranged as

$$
\begin{gathered}
\frac{\partial^{\alpha} u(x, t)}{\partial t^{\alpha}}=\frac{1}{\Gamma(1-\alpha)} \int_{0}^{t}(t-s)^{-\alpha}\left[x_{l}\left[\dot{\mathrm{g}}_{1}\left(t_{s+1}\right)-\dot{\mathrm{g}}_{0}\left(t_{s+1}\right)\right]\right. \\
\left.+\dot{\mathrm{g}}_{0}\left(t_{s+1}\right)\right] d s+\frac{1}{\Gamma(1-\alpha)}\left[\boldsymbol{C}_{(m)}^{T} \boldsymbol{P}_{(m)}^{2} \boldsymbol{h}_{(m)}(x)\right. \\
\left.-x_{l} \boldsymbol{C}_{(m)}^{T} \boldsymbol{P}_{(m)}^{2} \boldsymbol{f}\right]\left(\frac{t^{1-\alpha}}{1-\alpha}\right)
\end{gathered}
$$

And $\frac{\partial^{1-\alpha}}{\partial t^{1-\alpha}}\left[\frac{\partial^{2} u(x, t)}{\partial x^{2}}\right]$ can be calculated as

$$
\begin{gathered}
\frac{\partial^{1-\alpha}}{\partial t^{1-\alpha}}\left[\frac{\partial^{2} u(x, t)}{\partial x^{2}}\right]=\frac{\partial^{\alpha-1} u(x, t)}{\partial t^{\alpha-1}}\left[u^{\prime \prime}\left(x, t_{s}\right)+(t\right. \\
\left.\left.-t_{s}\right) \boldsymbol{C}_{(m)}^{T} \boldsymbol{h}_{(m)}(x)\right] \\
=\frac{1}{\Gamma(1-(1-\alpha))} \int_{0}^{t}(t-s)^{-(1-\alpha)} \frac{d}{d s}\left[u^{\prime \prime}\left(x, t_{s}\right)\right. \\
\left.+\left(s-t_{s}\right) \boldsymbol{C}_{(m)}^{T} \boldsymbol{h}_{(m)}(x)\right] d s
\end{gathered}
$$




$$
\begin{gathered}
=\frac{1}{\Gamma(\alpha)} \int_{0}^{t}(t-s)^{-(1-\alpha)}\left[\boldsymbol{C}_{(m)}^{T} \boldsymbol{h}_{(m)}(x)\right] d s \\
=\frac{-t}{\alpha \Gamma(\alpha)} \boldsymbol{C}_{(m)}^{T} \boldsymbol{h}_{(m)}(x)
\end{gathered}
$$

then by Caputo definition of fractional derivative and equation (34) we get

$$
\begin{array}{r}
\frac{\partial^{1-\alpha}}{\partial t^{1-\alpha}} \int_{0}^{x} u(s, \mathrm{t}) d s=\frac{\mathrm{t}^{\alpha}}{\Gamma(1+\alpha)} \boldsymbol{C}_{(m)}^{T} \boldsymbol{P}_{(m)}^{3} \boldsymbol{h}_{(m)}\left(x_{l}\right)+x_{l} \dot{\mathrm{g}}_{0}\left(t_{s+1}\right) \\
\frac{\partial u(x, t)}{\partial \mathrm{t}}=\frac{\partial^{1-\alpha}}{\partial t^{1-\alpha}} u^{\prime \prime}\left(x_{l}, t_{s+1}\right)+\sigma \frac{\partial^{1-\alpha}}{\partial t^{1-\alpha}} \int_{0}^{x} u(s, t) d s+\frac{\partial^{1-\alpha}}{\partial t^{1-\alpha}} f\left(x_{l}, t_{s+1}\right)
\end{array}
$$

The Haar coefficients vector $C_{(m)}$ are calculated from the system of linear equations (39) or (40). The solution is found according to equation (32).


Figure 3. Comparison between the exact solution and the approximate solutions of the partial differential equation for Case 3 equation (46) for $m=$ $8,16,32,64$ with $\alpha=0.9$.
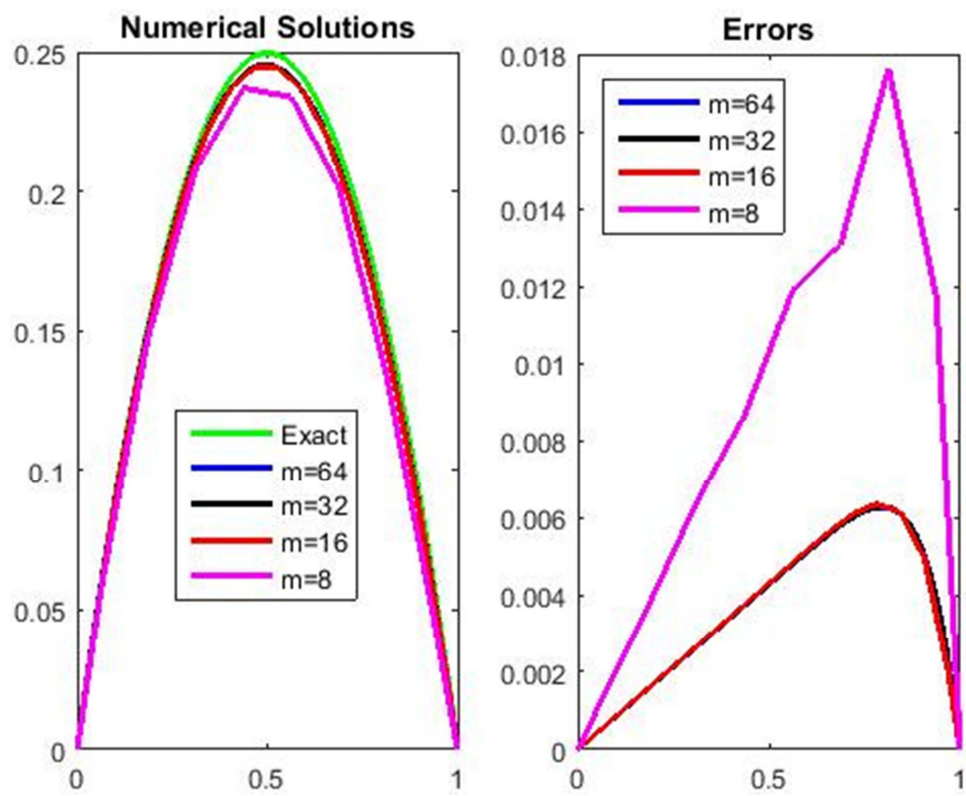

Figure 4. Solution and the approximate solutions of the partial differential equation for Case 3 equation (46) for $m=8,16,32,64 \mathrm{with} \alpha=0.7$. 


\section{The Average Error}

It is generally accepted that the error is understood as the difference between the exact and the calculated solutions. The error can be calculated at any point, let us denote by $\Delta(l)$ to the error at the point $\left(\frac{2 l-1}{2 m}, T_{\text {fin }}\right)$ so

$$
\Delta(l)=u_{e x}\left(\frac{2 l-1}{2 m}, T_{\text {fin }}\right)-u\left(\frac{2 l-1}{2 m}, T_{\text {fin }}\right), l=1,2,3, \cdots, m
$$

The maximum error $M_{\Delta}$ is defined as

$$
M_{\Delta}=\operatorname{Max}|\Delta(l)|
$$

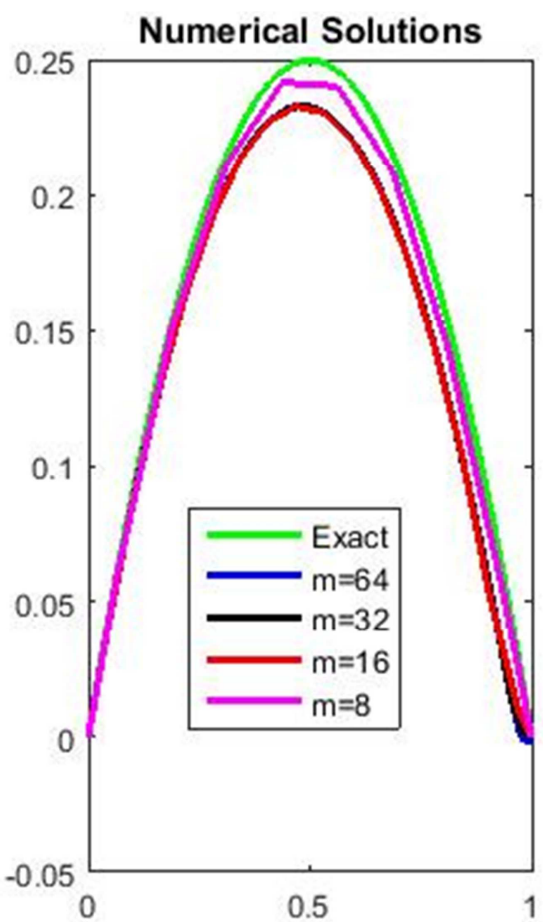

The average error $\sigma_{\Delta}$ can be defined as

$$
\sigma_{\Delta}=\frac{\sum_{l=1}^{l=m}|\Delta(l)|}{m}
$$

Increasing number of collocation points not always give a better solution, in some cases by increasing the number of collocation points the coefficient matrix may turn out to be nearly singular, this increase error in coefficients matrix, for more details we recomined $[6,17,18]$.

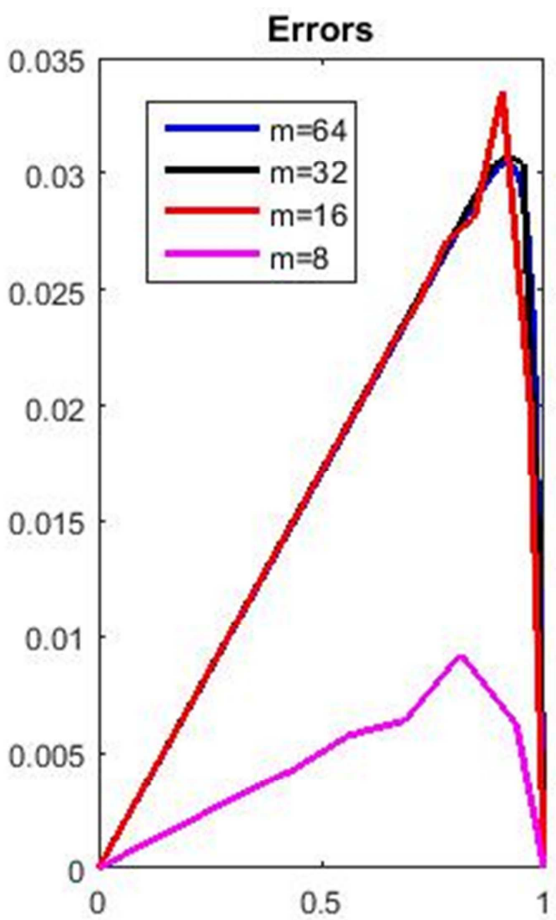

Figure 5. Comparison between the exact solution and the approximate solutions of the partial differential equation for Case 3 equation (51) for $m=$ $8,16,32,64$ with $\alpha=0.9$.

\section{Numerical Example}

We consider equation (2) with initial condition $u(x, 0)=$ $\mathrm{x}-x^{2}$ and homogeneous boundary conditions $u(0, t)=$ $u(1, t)=0$. In order to recognize the effect of the different terms we divided the problem to four cases and the force term $f(x, t)$ is used to adopt the exact solution to be $u(x, t)=$ $\left(x-x^{2}\right) e^{-t}$. All results are given with $\Delta x=0.1, \Delta t=0.0001$ and $T_{\text {fin }}=0.001$

\subsection{Case 1}

The classical (integer) diffusion equation with non homogenous term $\sigma=0, \alpha=1$, then equation (2) take the form

$$
\frac{\partial u(x, t)}{\partial t}=\frac{\partial^{2} u(x, t)}{\partial x^{2}}+\left(x^{2}-x+2\right) e^{-t}
$$

Then equation (33) will be

$$
\dot{u}\left(x_{l}, t_{s}\right)=\boldsymbol{C}_{(m)}^{T} \boldsymbol{P}_{(m)}^{2} \boldsymbol{h}_{(m)}(x)-x_{l} \boldsymbol{C}_{(m)}^{T} \boldsymbol{P}_{(m)}^{2} \boldsymbol{f}
$$

Substitute equations (42) and (30) in equation (41) we obtain $\boldsymbol{C}_{(m)}^{T} \boldsymbol{P}_{(m)}^{2} \boldsymbol{h}_{(m)}(x)-x_{l} \boldsymbol{C}_{(m)}^{T} \boldsymbol{P}_{(m)}^{2} \boldsymbol{f}-d t \boldsymbol{C}_{(m)}^{T} \boldsymbol{h}_{(m)}(x)$

$$
-u^{\prime \prime}\left(x_{l}, t_{s}\right)-e^{-t_{s+1}}\left(x_{l}^{2}-\mathrm{x}_{l}+2\right)=0
$$

and equation (3) take the same form of equation (2)

\subsection{Case 2}

The classical (integer) diffusion equation with space dependent integral term and non homogenous term, $\sigma=1$, $\alpha=1$ then equation (2) take the form

$\frac{\partial u(x, t)}{\partial t}=\frac{\partial^{2} u(x, t)}{\partial x^{2}}+\int_{0}^{x} u(\mathrm{~s}, t) d s+\left(\frac{x^{3}}{3}+\frac{x^{2}}{2}-\mathrm{x}+2\right) e^{-t}$

Calculate equation (34) and Substitute it with equations (42) and (30) in equation (44) to get 


$$
\begin{array}{r}
C_{(m)}^{T} P_{(m)}^{2} h_{(m)}(x)-x_{l} C_{(m)}^{T} P_{(m)}^{2} f-d t C_{(m)}^{T} h_{(m)}(x)-\operatorname{dt} C_{(m)}^{T} P_{(m)}^{3} h_{(m)}(x) \\
-\left(\frac{x^{3}}{3}+\frac{\mathrm{x}^{2}}{2}-\mathrm{x}+2\right) e^{-t_{S+1}}-u^{\prime \prime}\left(x_{l}, t_{s}\right)+\mathrm{x}_{l}^{2} \mathrm{dt} C_{(m)}^{T} P_{(m)} f-x_{l} u\left(x_{l}, t_{s}\right)=0
\end{array}
$$

and equation (3) take the same form of equation (2).
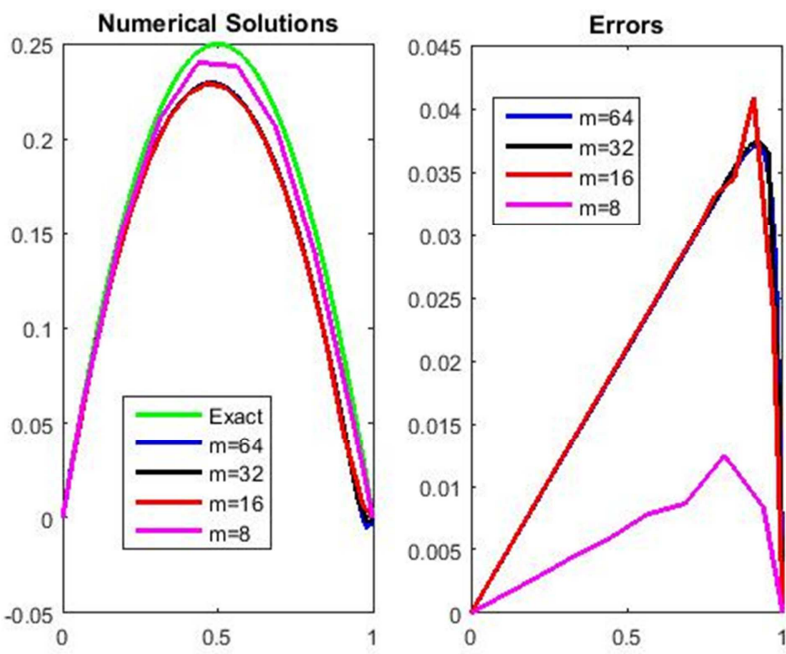

Figure 6. Comparison between the exact solution and the approximate solutions of the partial differential equation for Case 3 equation (49) for $m=8,16,32,64$ with $\alpha=0.7$.

\subsection{Case 3}

The fractional time diffusion equation, when $\sigma=0$, $0<\alpha \leq 1$ then equation (2) take the form

$$
\frac{\partial^{\alpha} u(x, t)}{\partial t^{\alpha}}=\frac{\partial^{2} u(x, t)}{\partial x^{2}}+\left(x-x^{2}\right) \frac{\partial^{\alpha}}{\partial t^{\alpha}} e^{-t}-2 e^{-t}
$$

Then equation (36) will be

$$
\frac{\partial^{\alpha} u(x, t)}{\partial t^{\alpha}}=\frac{1}{\Gamma(1-\alpha)}\left[\boldsymbol{C}_{(m)}^{T} \boldsymbol{P}_{(m)}^{2} \boldsymbol{h}_{(m)}(x)-x_{l} \boldsymbol{C}_{(m)}^{T} \boldsymbol{P}_{(m)}^{2} \mathbf{f}\right]\left(\frac{t^{1-\alpha}}{1-\alpha}\right)
$$

Substitute equations (47) and (30) in equation (46) we obtain

$$
\begin{aligned}
& \frac{1}{\Gamma(1-\alpha)}\left[\boldsymbol{C}_{(m)}^{T} \boldsymbol{P}_{(m)}^{2} \boldsymbol{h}_{(m)}(x)-x_{l} \boldsymbol{C}_{(m)}^{T} \boldsymbol{P}_{(m)}^{2} \mathbf{f}\right]\left(\frac{t^{1-\alpha}}{1-\alpha}\right)-2 e^{-t_{s+1}} \\
& -\boldsymbol{d t} \boldsymbol{C}_{(m)}^{T} \boldsymbol{h}_{(m)}(x)-\mathrm{u}^{\prime \prime}\left(\mathrm{x}_{l}, \mathrm{t}_{s}\right)-\left(x_{l}-x_{l}^{2}\right) \frac{\partial^{\alpha}}{\partial t^{\alpha}} e^{-t_{s+1}}=0
\end{aligned}
$$

and equation (3) take the form

$$
\frac{\partial u(x, t)}{\partial t}=\frac{\partial^{1-\alpha}}{\partial t^{1-\alpha}}\left(\frac{\partial^{2} u(x, t)}{\partial x^{2}}\right)-\left(x-x^{2}\right) e^{-t}-2 \frac{\partial^{1-\alpha}}{\partial t^{1-\alpha}} e^{-t}
$$

Substitute equations (42) and (34) in equation (49), it is found

$$
\begin{gathered}
\boldsymbol{C}_{(m)}^{T} \boldsymbol{P}_{(m)}^{2} \boldsymbol{h}_{(m)}\left(x_{l}\right)-x_{l} \boldsymbol{C}_{(m)}^{T} \boldsymbol{P}_{(m)}^{2} \boldsymbol{f}-\frac{t_{s+1}}{\alpha \Gamma(\alpha)} \boldsymbol{C}_{(m)}^{T} \boldsymbol{h}_{(m)}\left(x_{l}\right) \\
+\left(x_{l}-x_{l}^{2}\right) e^{-t_{S+1}}-2 \frac{\partial^{1-\alpha}}{\partial t^{1-\alpha}} e^{-t_{S+1}}=0
\end{gathered}
$$$$
\boldsymbol{C}_{(m)}^{T} \boldsymbol{P}_{(m)}^{2} \boldsymbol{h}_{(m)}\left(x_{l}\right)-x_{l} \boldsymbol{C}_{(m)}^{T} \boldsymbol{P}_{(m)}^{2} \boldsymbol{f}+x_{l}^{2} \frac{t^{\alpha}}{\Gamma(1+\alpha)} \boldsymbol{C}_{(m)}^{T} \boldsymbol{P}_{(m)} \boldsymbol{f}-\frac{t}{\alpha \Gamma(\alpha)} \boldsymbol{C}_{(m)}^{T} \boldsymbol{h}_{(m)}\left(x_{l}\right)
$$$$
-\frac{t^{\alpha}}{\Gamma(1+\alpha)} \boldsymbol{C}_{(m)}^{T} \boldsymbol{P}_{(m)}^{3} \boldsymbol{h}_{(m)}\left(x_{l}\right)+\left(x_{l}-x_{l}^{2}\right) e^{-t_{s+1}}+\left(\frac{x^{2}}{2}-\frac{x^{3}}{3}-2\right) \frac{\partial^{1-\alpha}}{\partial t^{1-\alpha}} e^{-t_{s+1}}=0
$$
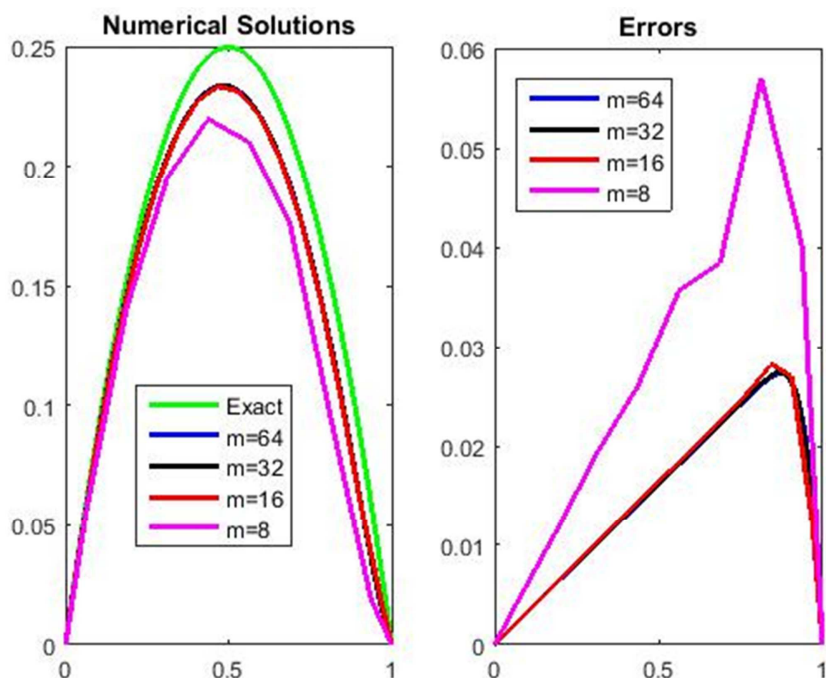

Figure 7. Comparison between the exact solution and the approximate solutions of the partial differential equation for Case 4 equation (51) for $=8,16,32,64 \alpha=0.9$.

\subsection{Case 4}

The fractional time diffusion equation, when $\sigma=1$, $0<\alpha \leq 1$ then equation (2) take the form

$$
\begin{gathered}
\frac{\partial^{\alpha} u(x, t)}{\partial t^{\alpha}}=\frac{\partial^{2} u(x, t)}{\partial x^{2}}+\int_{0}^{t} u(s, t) d s+\left(x-x^{2}\right) \frac{\partial^{\alpha}}{\partial t^{\alpha}} e^{-t} \\
-\left(\frac{x^{2}}{2}-\frac{x^{3}}{3}-2\right) e^{-t}
\end{gathered}
$$

Use equation (34) with equations (47), (30) and substitute them in equation (51) to get

$$
\begin{gathered}
\frac{1}{\Gamma(1-\alpha)}\left(\boldsymbol{C}_{(m)}^{T} \boldsymbol{P}_{(m)}^{2} \boldsymbol{h}_{(m)}\left(x_{l}\right)-x_{l} \boldsymbol{C}_{(m)}^{T} \boldsymbol{P}_{(m)}^{2} \boldsymbol{f}\right)\left(\frac{t_{s+1}^{1-\alpha}}{1-\alpha}\right) \\
-\mathrm{u}^{\prime \prime}\left(\mathrm{x}_{l}, \mathrm{t}_{s}\right)-\operatorname{dt} \boldsymbol{C}_{(m)}^{T} \boldsymbol{h}_{(m)}\left(x_{l}\right)+x_{l}^{2} \mathrm{dt} \boldsymbol{C}_{(m)}^{T} \boldsymbol{P}_{(m)} \boldsymbol{f} \\
-\operatorname{dt} \boldsymbol{C}_{(m)}^{T} \boldsymbol{P}_{(m)}^{3} \boldsymbol{h}_{(m)}\left(x_{l}\right)-x_{l} u\left(x_{l}, t_{s}\right) \\
+\left(\frac{x^{2}}{2}-\frac{x^{3}}{3}-2\right) e^{-t_{s+1}}-\left(\mathrm{x}_{l}-\mathrm{x}_{l}^{2}\right) \frac{\partial^{\alpha}}{\partial t^{\alpha}} e^{-t_{s+1}}
\end{gathered}
$$

and equation (3) take the form

$$
\begin{gathered}
\frac{\partial u(x, t)}{\partial t}=\frac{\partial^{1-\alpha}}{\partial t^{1-\alpha}}\left(\frac{\partial^{2} u(x, t)}{\partial x^{2}}\right)+\frac{\partial^{1-\alpha}}{\partial t^{1-\alpha}} \int_{0}^{x} u(\mathrm{~s}, \mathrm{t}) d s \\
-\left(\frac{x^{2}}{2}-\frac{x^{3}}{3}-2\right) \frac{\partial^{1-\alpha}}{\partial t^{1-\alpha}} e^{-t_{s+1}}-\left(\mathrm{x}_{l}-\mathrm{x}_{l}^{2}\right) e^{-t_{s+1}}
\end{gathered}
$$

Use equation (42) with equations (33) and (34) and substitute them in equation (53) to get $t^{\alpha}$ 

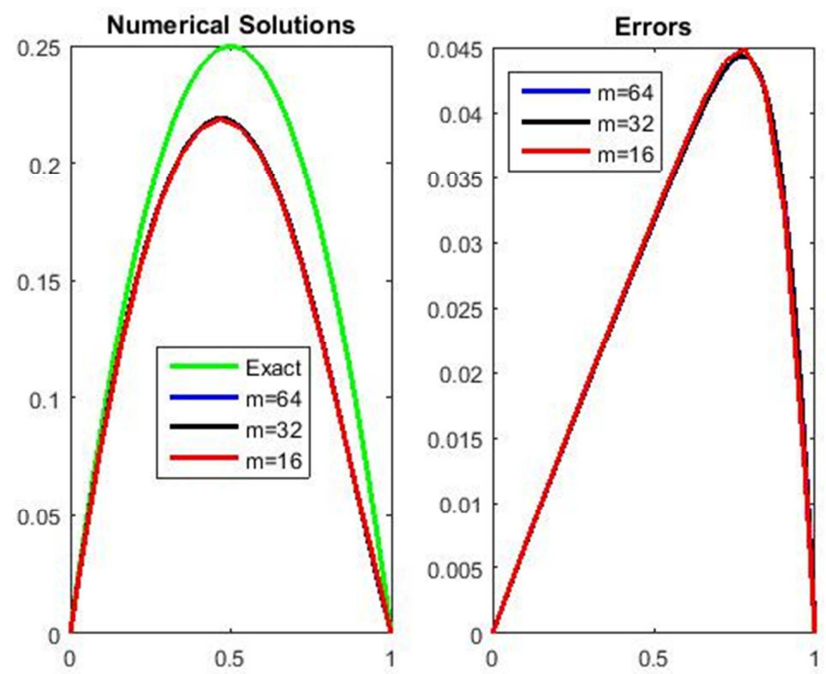

Figure 8. Comparison between the exact solution and the approximate solutions of the partial differential equation for Case 4 equation (51) for $m=16,32,64$ with $\alpha=0.7$ ( $m=8$ is unstable).

\section{Conclusion}

The use of memory terms in the form of fractional derivatives or integral forms is step towards realistic mathematical models. The fractional time derivative considers the memory and hereditary behaviours, while the integral terms considers the global effects of the variable under consideration (space). The use of inverse operator has improved the results significantly due to the memory effects of the fractional time derivatives which are extended to all other terms in the equation and affects on the global terms appears in the integral forms instead of only three terms (integer space derivatives). The use of operational matrix has facilitated the evaluation of the complicated integrals. In the numerical calculation section the complete problem is divided into four cases ranging from the classical integer case up to the fractional order with space dependent integral term.
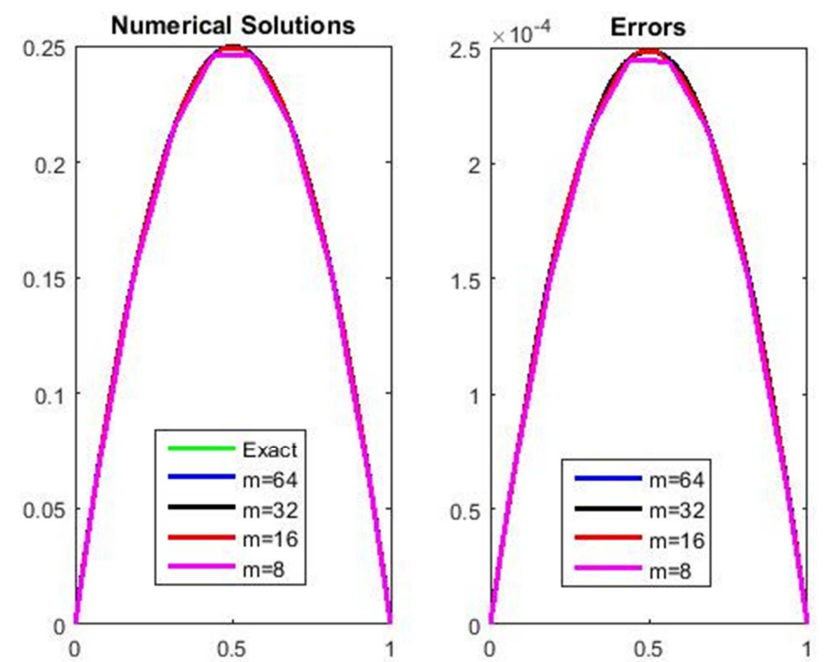

Figure 9. Comparison between the exact solution and the approximate solutions of the partial differential equation for Case 4 equation (53) for $m=8,16,32,64$ with $\alpha=0.9$.
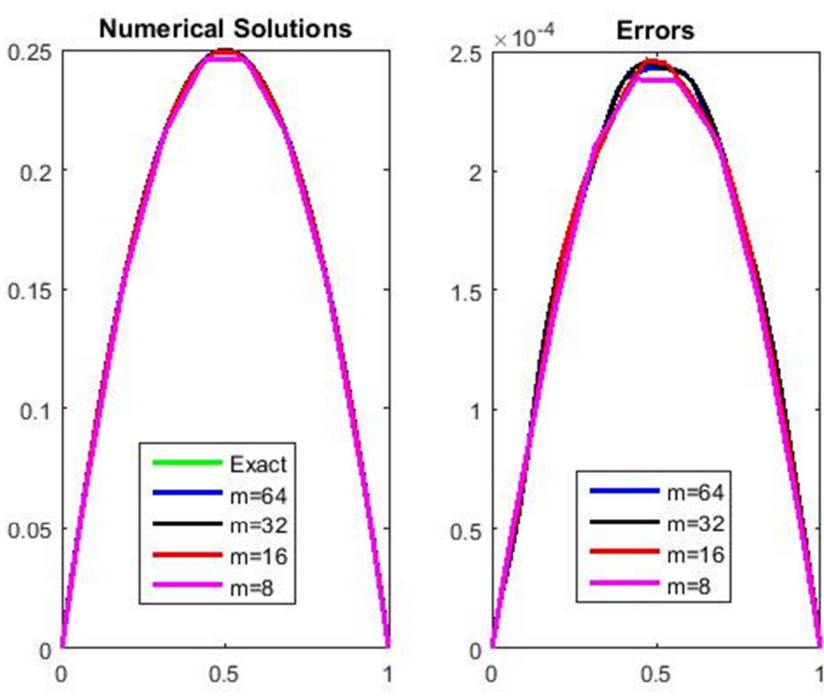

Figure 10. Comparison between the exact solution and the approximate solutions of the partial differential equation for Case 4 equation (53) for $m=8,16,32,64$ with $\alpha=0.7$.

The calculated results illustrate that the wavelet techniques can be applied to many other problems. Recently, many modification in using different bases functions is used to increase the accuracy as required. and this will be our objective in a subsequent work.

The numerical calculations illustrated the reliability of the wavelet technique in solving PDE as shown in the figures from 1 to 10. Also, the use of the inverse operator has moved the memory effects appears in fractional derivatives to the overall domain.

\section{References}

[1] E. M. E. Elbarbary, S. M. Elsayed, I. K. Youssef, A. M. Khodier, Restrictive Chebyshev rational approximation and applications to heat-conduction problems, Applied Mathematics and Computation 136 (2-3) (2003) 395-403.

[2] K. Rektorys, The Method of Discretization in Time and Partial Differential Equations, Mathematics and its Applications, Springer Netherlands, 1982.

[3] A. M. A. El-Sayed, Fractional-order diffusion-wave equation, International Journal of Theoretical Physics, Springer Netherlands, 35 (2) (1996) 311-322.

[4] I. K. Youssef, A. M. Shukur, The line method combined with spectral chebyshev for space-time fractional diffusion equation, Applied and Computational Mathematics 3 (6) (2014) 330-336.

[5] I. K. Youssef, A. M. Shukur, Modified variation iteration method for fraction space-time partial differential heat and wave equations, International Journal 2 (2) (2013) 1000-1013.

[6] I. K. Youssef, A. R. A. Ali, Memory Effects in Diffusion Like Equation Via Haar Wavelets, Pure and Applied Mathematics Journal, 5 (4), (2016) 130-140.

[7] I. Podlubny, Fractional Differential Equations, Camb. Academic Press, San Diego, CA, 1999. 
[8] K. Miller, B. Ross, An Introduction to the Fractional Calculus and Fractional Differential Equations, New York: Wiley-Interscience Publ., 1993.

[9] Ü. Lepik, Solving fractional integral equations by the haar wavelet method, Applied Mathematics and Computation 214 (2) (2009) 468-478.

[10] I. K. Youssef, A. M. Shukur, Precondition for discretized fractional boundary value problem, Pure and Applied Mathematics Journal 3 (1) (2014) 1-6.

[11] G. D. Smith, Numerical Solution of Partial Differential Equations Finite Difference Methods, Oxford University Press, 1978.

[12] C. K. Chui, An introduction to wavelets, Vol. 1, Academic press, 2014

[13] Ü. Lepik, Buckling of elastic beams by the haar wavelet method, Estonian Journal of Engineering 17 (3) (2011) 271284.

[14] C. F. Chen, C. H. Hsiao, Haar wavelet method for solving lumped and distributed-parameter systems, IEE Proc., Control Theory Appl. 144 (1) (1997) 87-94. doi: 10.1049/ip-cta: 19970702.
[15] C. F. Chen, C. H. Hsiao, Wavelet approach to optimising dynamic systems, IEE Proc., Control Theory Appl. 146 (2) (1999) 213-219. doi: 10.1049/ip-cta:19990516.

[16] Ü. Lepik, Application of the haar wavelet transform to solving integral and differential equations, Proc. Estonian Acad. Sci. Phys. Math. 56 (1) (2007) 28-46.

[17] Ü. Lepik, Numerical solution of differential equations using haar wavelets, Mathematics and computers in simulation 68 (2) (2005) 127-143.

[18] Ü. Lepik, Numerical solution of evolution equations by the haar wavelet method, Applied Mathematics and Computation 185 (1) (2007) 695-704.

[19] C. H. Hsiao, Haar wavelet direct method for solving variational problems, Mathematics and Computers in Simulation 64 (5) (2004) 569-585.

[20] C. H. Hsiao, W. J. Wang, Haar wavelet approach to nonlinear stiff systems, Mathematics and computers in simulation 57 (6) (2001) 347-353. 